
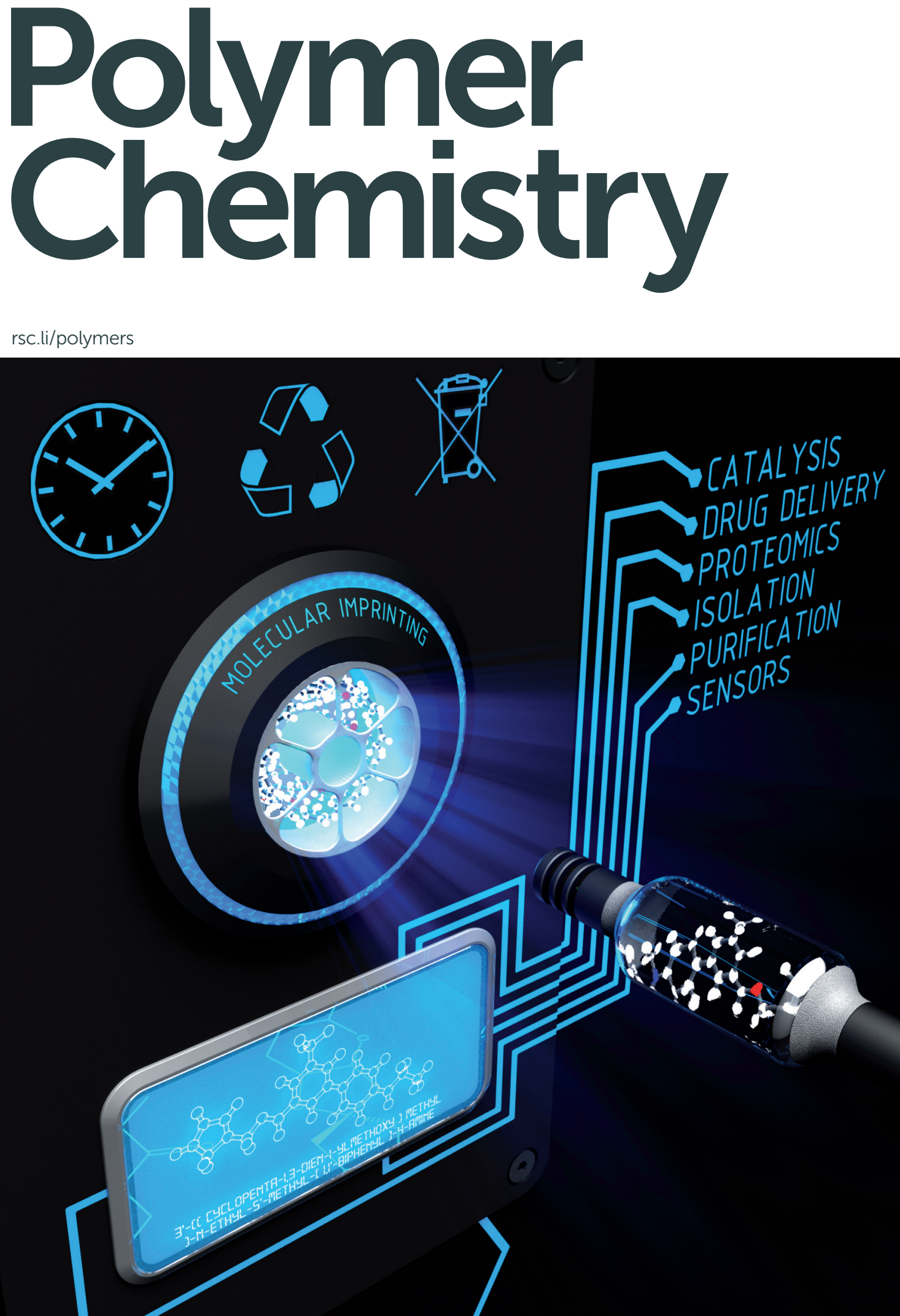

ISSN 1759-9962

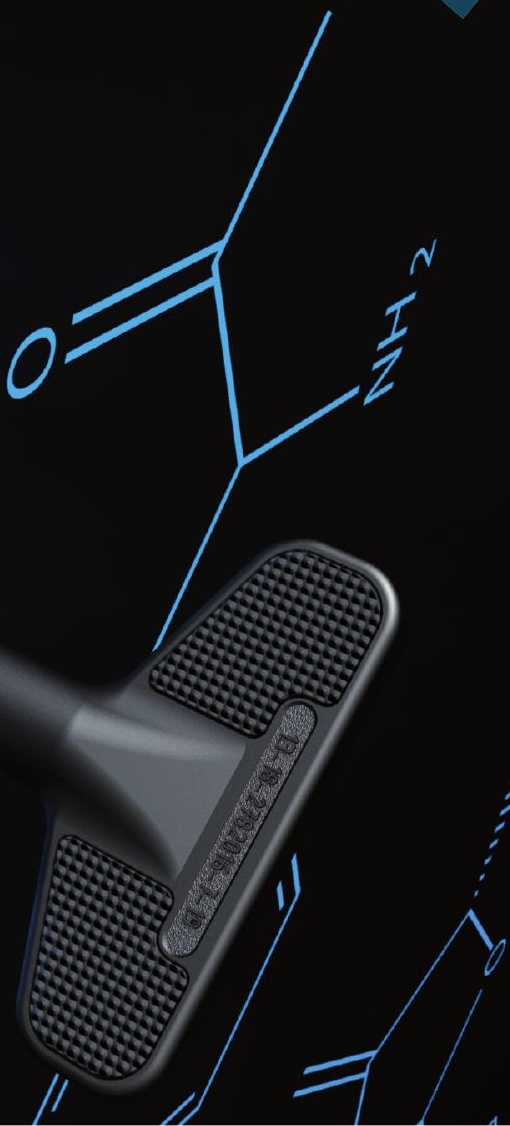

ISSN 1759-9962

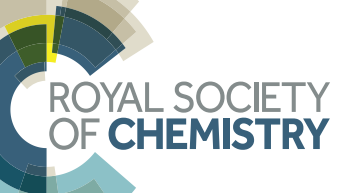




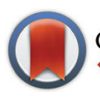

CrossMark \&lick for updates

Cite this: Polym. Chem., 2017, 8, 666

\title{
Long-term stability and reusability of molecularly imprinted polymers $\uparrow$
}

\author{
Jozsef Kupai, ${ }^{\text {a,b }}$ Mayamin Razali, ${ }^{a}$ Sibel Buyuktiryaki, ${ }^{\mathrm{c}}$ Rustem Kecili ${ }^{\mathrm{c}}$ and \\ Gyorgy Szekely*a
}

\begin{abstract}
Molecularly imprinted materials are man-made mimics of biological receptors. Their polymer network has recognition sites complementary to a substrate in terms of size, shape and chemical functionality. They have diverse applications in various chemical, biomedical and engineering fields such as solid phase extraction, catalysis, drug delivery, pharmaceutical purification, (bio)sensors, water treatment, membrane separations and proteomics. The stability and reusability of molecularly imprinted polymers (IPs) have crucial roles in developing applications that are reliable, economic and sustainable. In the present article the effect of crosslinkers, functional monomers and conditions for template extraction on the long-term stability and reusability of IPs was systematically investigated. Adsorption capacity, selectivity, morphology and thermal decomposition of eleven different L-phenylalanine methyl ester imprinted polymers were studied to reveal performance loss over 100 adsorption-regeneration cycles. Furthermore, crosslinker and functional monomer specific reversible and irreversible decomposition of imprinted polymers as a result of adsorbent regeneration were investigated through adsorption studies, electron microscopy, $\mathrm{N}_{2}$ adsorption and thermogravimetric analysis. A decomposition mechanism was proposed and revealed using NMR spectroscopy. Solutions to avoid or overcome the limitations of the most common crosslinkers, functional monomers and extraction techniques were proposed and experimentally validated.
\end{abstract}

Received 21st October 2016 Accepted 23rd November 2016 DOI: $10.1039 / c 6 p y 01853 j$ www.rsc.org/polymers membranes and proteomics. ${ }^{2-7}$ Apart from the single-use analytical devices most of the chemical and engineering applications require regeneration of the IPs to improve efficiency, economics and sustainability.

The reusability of imprinted materials has a crucial role in developing applications that are reliable, economic and sustainable. Nonetheless, reusability studies in the literature are limited to about 10 adsorption cycles and there is no assessment of long-term stability and reusability of imprinted polymers. Although the monomer-template assemblies and their stability have been thoroughly studied both theoretically and experimentally, ${ }^{8-12}$ the sole investigation into the stability of IPs was reported by Svenson et al. for a theophylline imprinted methacrylate based copolymer. ${ }^{13}$ It was demonstrated that exposure to acids (1-10 M HCl), bases $\left(5-25 \% \mathrm{NH}_{3}\right)$, autoclave treatment and elevated temperatures up to $150{ }^{\circ} \mathrm{C}$ over a period of 24 hours does not result in loss of affinity for the template. Despite its importance, the literature on template extraction itself is also scarce and intended to reduce template leaching. ${ }^{14-16}$

The present study systematically investigates the effect of crosslinkers, functional monomers and conditions for template extraction on the long-term stability and reusability of imprinted polymers (Fig. 1). Twenty-four-hour adsorptionregeneration cycles for more than a 100 times were carried out 


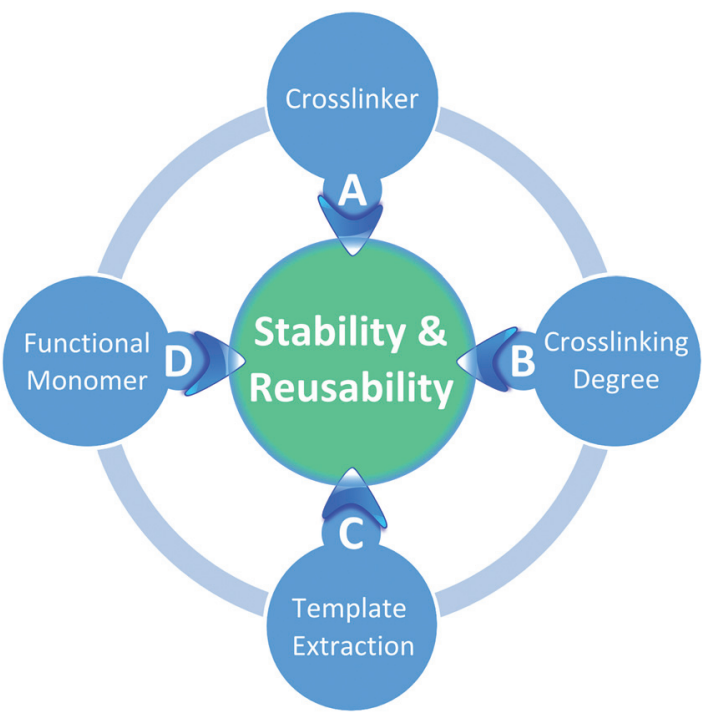

Fig. 1 The four main factors affecting the stability and reusability of molecularly imprinted polymers.

in duplicate, using eleven different L-phenylalanine methyl ester (ME) imprinted polymers having different compositions. Fig. 2 shows the structures of the template, functional monomers and crosslinkers employed in the present study. Although ME has biological relevance as it is the precursor and metabolite of the synthetic sweetener aspartame, ${ }^{17,18}$ the application of the prepared IPs is not the focal point of the present study. ME was chosen as the template because of its multifaceted chemical nature having hydrogen donor and<smiles>C=Cc1ccc(B(O)c2ccc(C(=C)C)cc2)c(C(=O)OCCOC(=O)C(=C)C(=O)OCCOC(=O)C(=C)C)c1</smiles><smiles>C=CC(=O)OC(C)(C)C</smiles><smiles>C=CC(=O)NCNC(=O)C=C</smiles>

Fig. 2 The L-phenylalanine methyl ester (ME) and benzyl ester (BE) were used as template and dummy template in this study. Ethylene glycol dimethacrylate (EDMA), divinylbenzene (DVB) and $N, N^{\prime}$-methylenebis(acrylamide) (BMA) were employed as cross-linkers whilst methacrylic acid (MA), acrylamide (AA), 1-(4-vinylphenyl)-3-(3,5-bis (trifluoromethyl)phenyl)urea (U), 4-vinylpyridine (VP), 4-vinylphenylboronic acid (PBA) and 4-vinylbenzoic acid (VBA) were used as functional monomers. acceptor sites as well as an aromatic moiety capable of $\pi-\pi$ interactions. Having such a versatile template in hand allowed investigating a wide range of functional monomers such as carboxylic acid, urea, amide, amine and boronic acid derivatives.

Acrylate, acrylamide and styrene based polymers were prepared using commonly employed crosslinkers such as ethylene glycol dimethacrylate (EDMA), divinylbenzene (DVB) and $N, N^{\prime}$ methylenebis(acrylamide) (BMA). The crosslinker is important in controlling the morphology of the polymer matrix, serves to stabilize the imprinted binding sites, and imparts mechanical stability to the polymer matrix in order to retain its molecular recognition capability. The crosslinking degree was set at 1/15 and 1/30 functional monomer to crosslinker ratios. The template extraction was carried out with washing solvents having different eluent strength at both room $\left(25^{\circ} \mathrm{C}\right)$ and elevated $\left(65^{\circ} \mathrm{C}\right)$ temperatures. Besides fundamental understanding, the present study aims to provide guidance on adsorbent regeneration for both professionals and academicians working in the field of polymers, in particular, imprinted materials.

\section{Experimental section}

\section{General}

Reagents (reagent grade) and solvents (analytical grade) were purchased from Sigma-Aldrich and Fisher Scientific, respectively. The 1-(4-vinylphenyl)-3-(3,5-bis(trifluoromethyl)phenyl) urea functional monomer was synthesized following the protocol reported by Hall et al., ${ }^{19}$ and its characterisation is included in the ESI. $\dagger$ Acetonitrile porogen was dried over $4 \AA$ molecular sieves. AIBN was purchased from Wako Pure Chemical Industry and purified by recrystallization from methanol. MA and EDMA were washed with $1 \mathrm{M}$ aqueous $\mathrm{NaOH}$ solution with distilled water, dried over anhydrous $\mathrm{Na}_{2} \mathrm{SO}_{4}$ and distilled over $\mathrm{CaH}_{2}$ under reduced pressure before use. A VWR-Hitachi Chromaster DAD-HPLC system equipped with an ACE $5 \mu \mathrm{m}, \mathrm{C} 18,300 \AA$, $250 \times 4.6 \mathrm{~mm}$ column was used for template analysis ( $260 \mathrm{~nm})$ with an isocratic elution using $25 \%$ acetonitrile and $75 \%$ phosphate buffer $(\mathrm{pH}=7)$. Elemental analyses were performed in the Microanalysis Laboratory of the School of Chemistry at The University of Manchester. The nitrogen sorption measurements were performed on a Quantachrome Autosorb 6B automatic adsorption instrument. Thermal gravimetric analysis (TGA) was performed with a Seiko Instrument Inc. TG-DTA 6200 using an alumina pan under a $50 \mathrm{~mL} \mathrm{~min}^{-1}$ nitrogen flow at a heating rate of $10{ }^{\circ} \mathrm{C} \mathrm{min}^{-1}$. NMR spectra were recorded in either $\mathrm{CDCl}_{3}$ or DMSO- $d_{6}$ on a Bruker DRX-500 Avance spectrometer (at $500 \mathrm{MHz}$ for ${ }^{1} \mathrm{H}$ and at $125 \mathrm{MHz}$ for ${ }^{13} \mathrm{C}$ spectra).

\section{Preparation of imprinted polymers}

IP microspheres were prepared by a suspension polymerisation method. ${ }^{20}$ Briefly, in a typical IP fabrication procedure $1 \mathrm{mmol}$ functional monomer, $1 \mathrm{mmol}$ template, $20 \mathrm{mmol}$ crosslinker, 0.1 wt $\%$ initiator, $100 \mathrm{mg}$ perfluoro polymeric surfactant 
Table 1 Stoichiometry and preparation conditions for the ME imprinted polymers. Corresponding control polymers (CP1-CP11) were prepared in the absence of template

\begin{tabular}{lllrr}
\hline Polymer & $\begin{array}{l}\text { Stoichiometry } \\
(\mathrm{mmol} / \mathrm{mmol} / \mathrm{mmol})\end{array}$ & $\begin{array}{l}\text { Porogen } \\
(\mathrm{mL})\end{array}$ & $\begin{array}{c}\text { PFPS } \\
(\mathrm{mg})\end{array}$ & $\begin{array}{r}\text { PMC } \\
(\mathrm{mL})\end{array}$ \\
\hline IP1 & ME/MA/EDMA (1/1/15) & 15 & 75 & 60 \\
IP2 & ME/MA/EDMA (1/1/30) & 15 & 150 & 120 \\
IP3 & ME/MA/DVB (1/1/15) & 15 & 75 & 60 \\
IP4 & ME/MA/DVB (1/1/30) & 15 & 150 & 120 \\
IP5 & ME/MA/BMA (1/1/15) & 15 & 75 & 60 \\
IP6 & ME/MA/BMA (1/1/30) & 15 & 150 & 120 \\
IP7 & ME/AA/DVB (1/1/15) & 15 & 75 & 60 \\
IP8 & ME/U/DVB (1/1/15) & 15 & 75 & 60 \\
IP9 & ME/VP/DVB (1/1/15) & 15 & 75 & 60 \\
IP10 & ME/PBA/DVB (1/1/15) & 15 & 75 & 60 \\
IP11 & ME/VBA/DVB (1/1/15) & 15 & 75 & 60 \\
& & & &
\end{tabular}

(PFPS) emulsifier, $80 \mathrm{~mL}$ perfluoro methylcyclohexane (PMC) dispersing phase and $15 \mathrm{~mL}$ acetonitrile porogen were stirred at $300 \mathrm{rpm}$ (Table 1). The imprinted polymers were obtained by polymerisation involving irradiation of the stirred mixture with UV light for 6 hours at a wavelength of $365 \mathrm{~nm}$ at room temperature under an inert atmosphere. The resulting beads were filtered and the remaining template and unreacted molecules were extracted by sequential two-hour Soxhlet extraction with methanol at $65{ }^{\circ} \mathrm{C}$. The IPs were dried under reduced pressure for $12 \mathrm{~h}$ at $25{ }^{\circ} \mathrm{C}$. Control polymers were prepared under identical conditions in the absence of a template. Different polymerisation techniques result in different morphologies that can affect the polymer degradation.

\section{Adsorption-regeneration cycles}

One adsorption-regeneration cycle consisted of loading the template, reaching equilibrium adsorption, followed by the extraction of the template and drying of the polymers. For the adsorption $200 \mathrm{~mL} 1 \mathrm{mM}$ template in acetonitrile was loaded per gram of adsorbent, which was then shaken at $300 \mathrm{rpm}$ at $25{ }^{\circ} \mathrm{C}$ for 24 hours in an incubator. The regeneration procedures under evaluation are compared in Table 2. Either solid phase extraction (SPE) or Soxhlet extractions (SXE) were used to remove the template and regenerate the polymers.

$120 \mathrm{~mL}$ of $0.1 \mathrm{M} \mathrm{HCl}$ or $\mathrm{NaOH}$ in methanol as washing solvents per gram of adsorbent were used for extraction. Either SPE cartridges at a washing flow rate of $1 \mathrm{~mL} \mathrm{~min}^{-1}$ at room temperature $\left(25^{\circ} \mathrm{C}\right)$ or two-hours SXE at the boiling point of

Table 2 Conditions for template extraction, i.e. polymer regeneration. Concentrations of hydrochloric acid and sodium hydroxide for the methanolic, aqueous solutions were $0.1 \mathrm{M}$

\begin{tabular}{lllll}
\hline$\#$ & Solvent & $T\left({ }^{\circ} \mathrm{C}\right)$ & $t(\mathrm{~h})$ & Method \\
\hline E1 & MeOH & 25 & 2 & SPE \\
E2 & MeOH & 65 & 2 & SXE \\
E3 & HCl in MeOH then water & 25 & 3 & SPE \\
E4 & HCl in MeOH then aq. HCl & 25 & 3 & SPE \\
E5 & NaOH in MeOH then water & 25 & 3 & SPE \\
E6 & NaOH in MeOH then aq. HCl & 25 & 3 & SPE
\end{tabular}

the solvent $\left(65^{\circ} \mathrm{C}\right)$ were used. The extraction was followed by a $100 \mathrm{~mL}$ methanol wash per gram of adsorbent at a flow rate of $1 \mathrm{~mL} \mathrm{~min}{ }^{-1}$ at room temperature in order to neutralise the polymers. 0.01 mbar vacuum pressure at room temperature was applied for 12 hours in order to dry the adsorbents. The experiments were carried out in duplicate with independently prepared polymers and the resulting standard deviations are shown in the adsorption diagrams.

\section{Results and discussion}

\section{Initial adsorption performance}

Removal of the template after the polymerisation process was monitored by HPLC and found to be between 91\% and 95\%. Afterwards continuous but negligible leaching of the template was observed below the limit of detection (LOD) of the analytical method. The initial absolute adsorption values for the first adsorption-regeneration cycle are shown in Fig. 3. Duplicate assays were carried out using independently prepared polymers and the values reported are averages of the two values obtained and standard deviations were between 1 and 5 for all assays. Rejection IP1, IP3 and IP5 featuring a functional monomer to crosslinker ratio of $1 / 15$ exhibited adsorption capacities of 185, 196 and $141 \mu \mathrm{mol}$ ME per gram of polymer, respectively. In accordance with the expectations corresponding IP2, IP4 and IP6 polymers with a double crosslinking degree, showed about halved (57\%, 56\% and 46\%) adsorption capacity. The imprinting factors were found to be between 2.9 and 5.2 proving successful incorporation of the template molecule into the polymer matrix during the polymerisation process. Polymers crosslinked at the same degree with EDMA and DVB showed similar adsorption capacities whilst BMA crosslinking at the lower crosslinking degree resulted in about 26\% lower values compared to EDMA and DVB crosslinking. The highest adsorption capacity and imprinting factor was demonstrated by IP3 comprising methacrylic acid (MA) as a functional monomer and DVB as a crosslinker. The polymers prepared with the other functional monomers showed $41-77 \%$ lower adsorption capacities (Fig. 3B).

\section{Adsorption performance in the long term}

In order to evaluate the long-term stability and reusability of the imprinted polymers adsorption-regeneration cycles were performed up to 100 times, and the results are summarized in Fig. 4 and 5 as relative adsorption compared to the initially obtained adsorption values (see Fig. 3). Duplicate assays were carried out using independently prepared polymers and the values reported are averages of the two values obtained and standard deviations were between 1 and 3 for all assays where no polymer degradation occurred. The conditions for regener-

$\ddagger$ The retention behaviour of imprinted polymers (IP) is often compared with the reference non-imprinted, control polymer (CP), to evaluate the effect of imprinting which is often expressed by the imprinting factor defined as the ratio of the adsorbed template by an IP and its corresponding CP. 

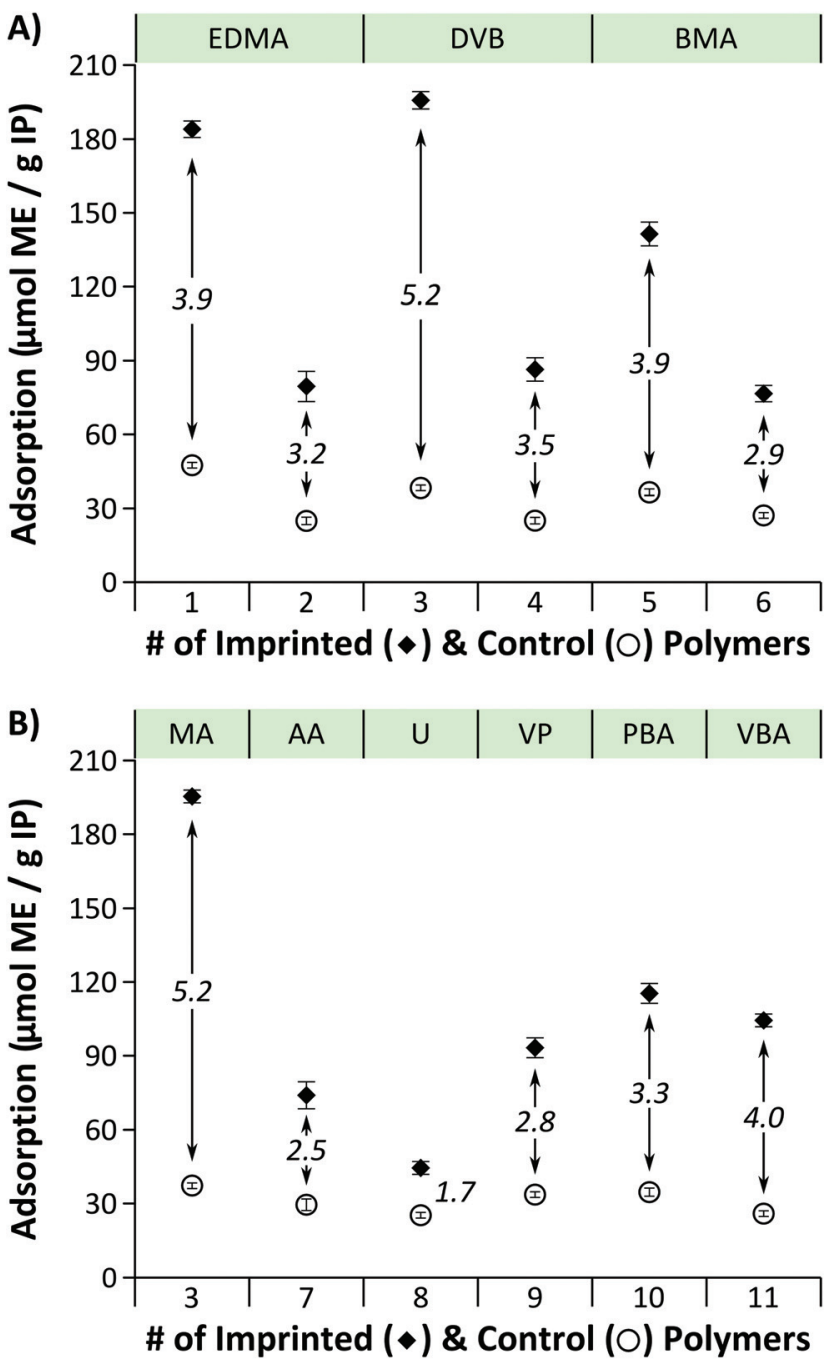

Fig. 3 Initial adsorption of L-phenylalanine methyl ester (ME). Panel (A) includes IP1-IP6 imprinted and control polymers demonstrating the effect of crosslinker and crosslinking degree on adsorption. Panel (B) includes IP3, IP7-IP11 imprinted and control polymers showing the effect of functional monomer on adsorption. See Table 1 for the polymer compositions. Imprinting factors

ation comprised of acidic, basic, room temperature and elevated temperature during extraction. It can be concluded that DVB crosslinking results in the most stable polymer irrespective of the degree of crosslinking. IP3 (1/15 MA/DVB) and IP4 (1/30 MA/DVB) can be reused at least 100 times without loss of affinity towards the template molecule using extraction methods E1 and E2 as shown in Fig. 4A and B, respectively. However, Fig. 4C and E reveal a relative adsorption decrease of about 5-15\% using extraction methods E3 and E5, respectively (see Table 2 for extraction conditions). These acidic and basic extractions were done in methanol. In contrast, extraction methods E4 and E6 included an additional step of aqueous $\mathrm{HCl}$ washing resulting in no performance loss of the polymers as depicted in Fig. 4D and F, respectively. The observed high stabilities of DVB crosslinked polymers are in accordance with the fact that stationary phases based on DVB have been successfully used for many years in reversed-phase high performance liquid chromatography due to their excellent chemical, $\mathrm{pH}$ and temperature stability. ${ }^{21}$

In contrast, the performance of EDMA and BMA crosslinked polymers deteriorates over time under both acidic (Fig. 4C and D) and basic (Fig. 4E and F) extraction conditions. The thermal stability profiles for IP1, IP3 and IP5 with the observations on adsorbent reusability are shown in Fig. 5. Whilst the $5 \%$ decomposition temperature values $\left(T_{\mathrm{d} 5}\right)$ for BMA and EDMA crosslinked polymers decreased by $14 \%$ (from $178^{\circ} \mathrm{C}$ to $153{ }^{\circ} \mathrm{C}$ ) and $24 \%$ (from $259^{\circ} \mathrm{C}$ to $197{ }^{\circ} \mathrm{C}$ ), respectively, the DVB-based polymer had the highest $T_{\mathrm{d} 5}$ value of $350^{\circ} \mathrm{C}$, which remained virtually the same. Fig. 6 shows how the morphology of the IP particles is affected by the regeneration method. The initial surface of all polymer particles is uniform (Fig. 6A) and remains the same even after 100 adsorption-regeneration cycles using the extraction method E2 (Fig. 6B).

However, the deterioration of IP1 and IP5 by the $100^{\text {th }}$ cycle resulted in an irregular surface with defects (Fig. 6C and D). A comparison of the surface areas for IP1, IP3 and IP5 shows the same trend (Fig. 7). The morphology of the DVB crosslinked polymer remains virtually the same over time, while the surface area for the EDMA- and BMA-based polymers monotonously decrease by $52 \%$ and $25 \%$ over 100 cycles, respectively. The decrease in surface area can be the result of the partial collapse of the 3D polymer network.

In general the relative adsorption can decrease as much as $50 \%$ by the $100^{\text {th }}$ adsorption-regeneration cycle. In all assays polymers having a higher crosslinking degree demonstrated higher stability and longer reusability. For instance IP2 (1/30 MA/EDMA) demonstrated about 10\% higher adsorption than IP1 (1/15 MA/EDMA) at the $100^{\text {th }}$ adsorption-regeneration cycle (Fig. 4C-F). Fig. 8 reveals the effect of the functional monomer on the reusability of imprinted polymers. AA and VP functional monomers in combination with the lower DVB crosslinking degree (1/15) yielded imprinted polymers (IP7 and IP9, respectively) which can be reused at least 100 times irrespective of the regeneration method (Fig. 8A-F). However, both acidic and basic conditions for template extraction using methods E3 and E5 (see Table 2) result in a performance loss of boronic acid (IP10) and carboxylic acid (IP3 and IP11) based polymers as shown in Fig. 8C and E. Nonetheless, incorporation of an aqueous $\mathrm{HCl}$ wash of the polymers resulted in significant improvement in performance for carboxylic acid based IP3 and IP11 (Fig. 8D and F). Both aliphatic and aromatic carboxylic acid polymers can be reused up to 100 adsorption-regeneration cycles using extraction methods E4 and E6.

On the other hand, the performance loss of boronic acid based IP10 can only be fully avoided with the extraction method E6, which requires basic conditions. An elevated temperature and basic regeneration conditions resulted in about $5 \%$ decrease in adsorption for the urea based IP8 by the $100^{\text {th }}$ adsorption-regeneration cycle as shown in Fig. 8B, E and F, respectively. These results are in accordance with the literature reporting that urea derivatives are more stable at acidic $\mathrm{pH}$ 

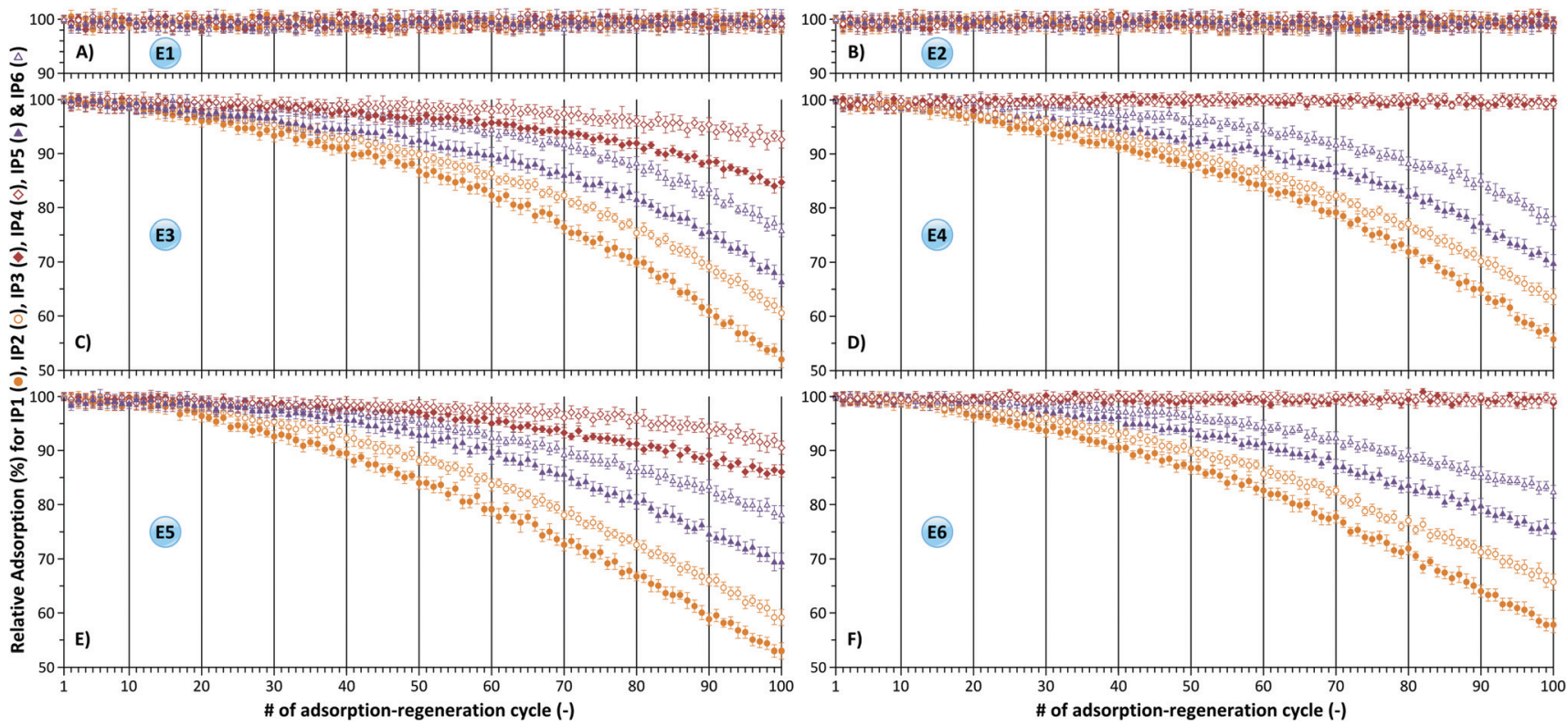

Fig. 4 Relative adsorption of ME on IP1-6 demonstrating the effect of crosslinker and crosslinking degree on the reusability of imprinted polymers. Template extraction was carried out using methods E1 (A), E2 (B), E3 (C), E4 (D), E5 (E) and E6 (F) in accordance with Table 2.

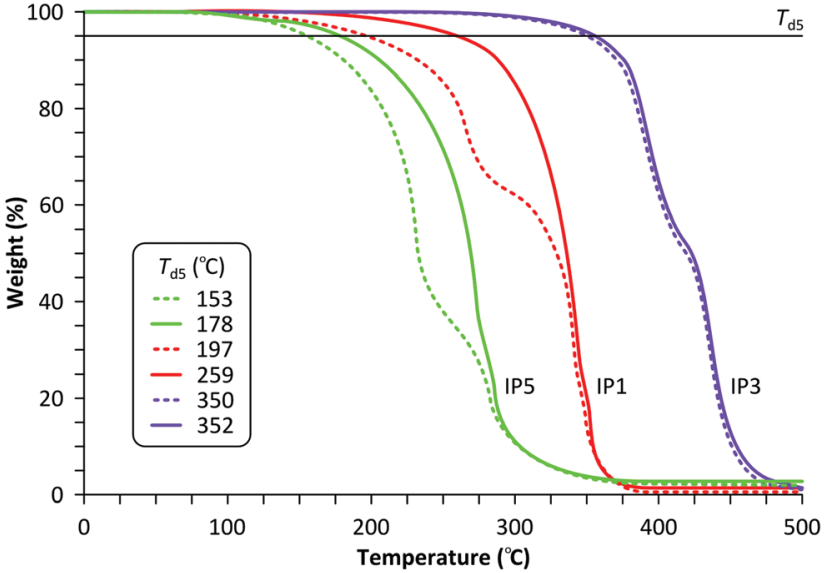

Fig. 5 TGA profiles and $5 \%$ decomposition temperature values $\left(T_{\mathrm{d} 5}\right)$ for BMA, EDMA and DVB crosslinked IP5, IP1 and IP3, respectively. Solid and dotted lines show the thermal stability after the $1^{\text {st }}$ and $100^{\text {th }}$ adsorption-regeneration cycle using $\mathrm{E} 6$, respectively.

and their stability decreases by increase in temperature for all pH values. ${ }^{22}$

The evaluation of imprinted polymer reusability in the literature is limited to 5-10 adsorption-regeneration cycles. ${ }^{23-25}$ However, these short-term studies can lead to hasty conclusions. The results of short-term studies cannot be used to draw conclusions on the long-term reusability of imprinted polymers. Interpretation of the very same data presented in Fig. 4 and 8 leads to different conclusions if solely the first 10 cycles are taken into account instead of the 100 cycles. In most cases long-term performance deterioration could not have been predicted merely based on the first 10 cycles.

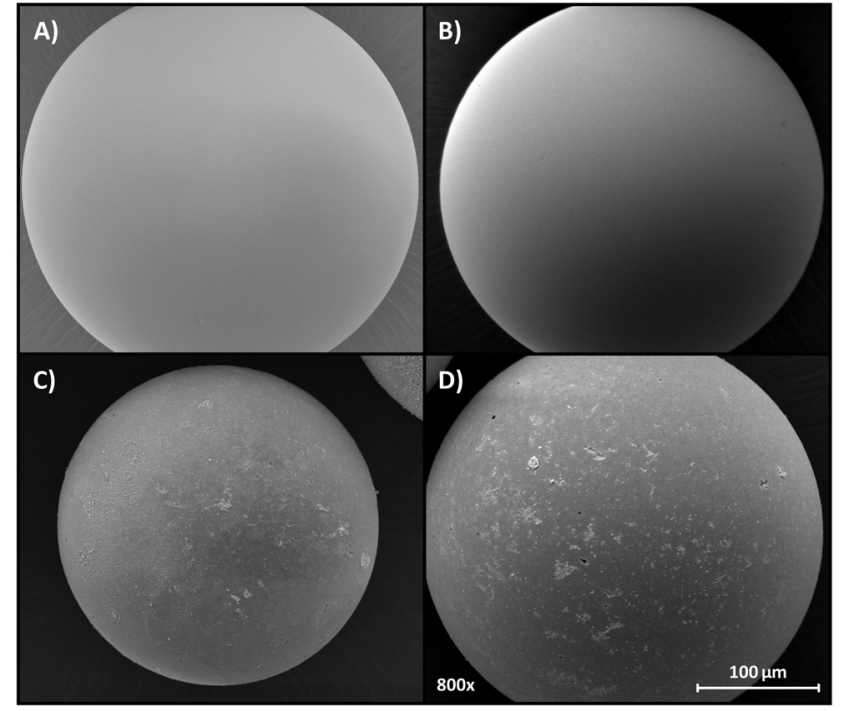

Fig. 6 Panel A shows a typical SEM image of freshly prepared IPs. Panel $B$ shows a typical SEM image of IP1, IP3 and IP5 microspheres after the $100^{\text {th }}$ cycle using the extraction method E2. Panels $C$ and $D$ reveal the deterioration of IP1 and IP5 after the $100^{\text {th }}$ cycle using the extraction method E6, respectively. See Table 2 for the regeneration methods.

The dummy template (BE) was used to investigate the selectivity of the polymers. Selectivity factors were calculated as the ratio of adsorbed $\mathrm{ME}$ and $\mathrm{BE}$, and the results are summarized in Fig. 9. In order to investigate whether the selectivity deteriorates in accordance with the loss of adsorption capacity, the boronic acid (IP10) and the carboxylic acid (IP11) based polymers were tested for BE adsorption at every $10^{\text {th }}$ adsorption-regeneration cycle. Proving successful incorporation of the template into the 


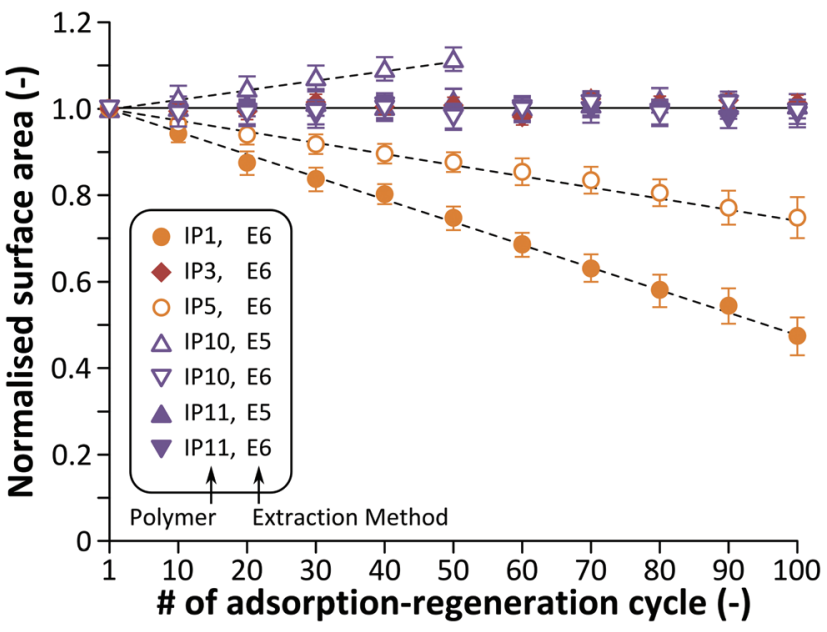

Fig. 7 Normalised surface area obtained by Brunauer-Emmett-Teller (BET) analysis. Using the extraction method E6 the surface area for IP1 and IP5 monotonously decreases while for IP3, IP10 and IP11 remains virtually constant over time. On the other hand, the surface area increases for IP10 when the extraction method E5 is employed. See Table $\mathrm{S} 2 \uparrow$ in the ESI for the absolute surface area values.

polymer matrix during the polymerisation process, selectivity factors of 6.5 and 5.2 were achieved during the first cycle for IP10 and IP11, respectively. Extraction methods E5 and E6 led to decreased and maintained selectivity, respectively.

These observations are in line with the changes in the adsorption capacity shown in Fig. $8 \mathrm{E}$ and F. Consequently, both selectivity and adsorption capacity of boronic and carboxylic acid based imprinted polymers can be maintained up to at least 100 adsorption-regeneration cycles with an acidic wash at the end of the regeneration process. From a morpho-

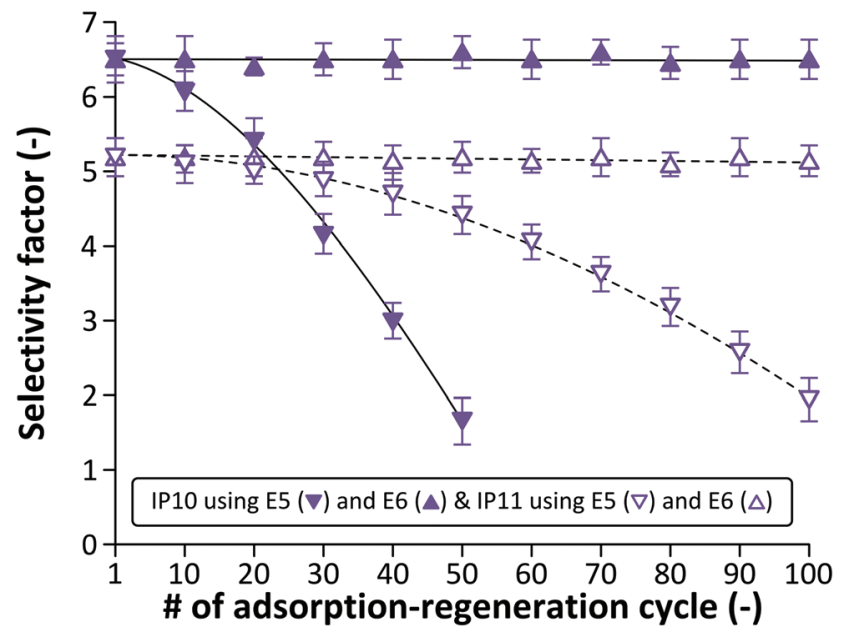

Fig. 9 Selectivity factors for boronic acid (IP10) and carboxylic acid (IP11) based polymers using extraction methods E5 and E6. The results demonstrate that an acid wash enables maintaining the initial selectivity of the polymers.

logical point Fig. 7 shows that the surface area for IP10 and IP11 remains virtually constant over time when the extraction method E6 is used (see Table S2 in the ESI $\dagger$ for the absolute values). However, without the acidic wash the surface area significantly increases: up to $11 \%$ by the $50^{\text {th }}$ cycle and $18 \%$ by the $100^{\text {th }}$ cycle. The increase in surface area is unexpected and it is probably due to the molecular level degradation of the polymer without collapse of the polymer network.

Leaching of the template and unreacted monomers from IPs is an often observed phenomenon, which is considered as one of the main drawbacks of the technology. ${ }^{15}$ They should be exhaus-
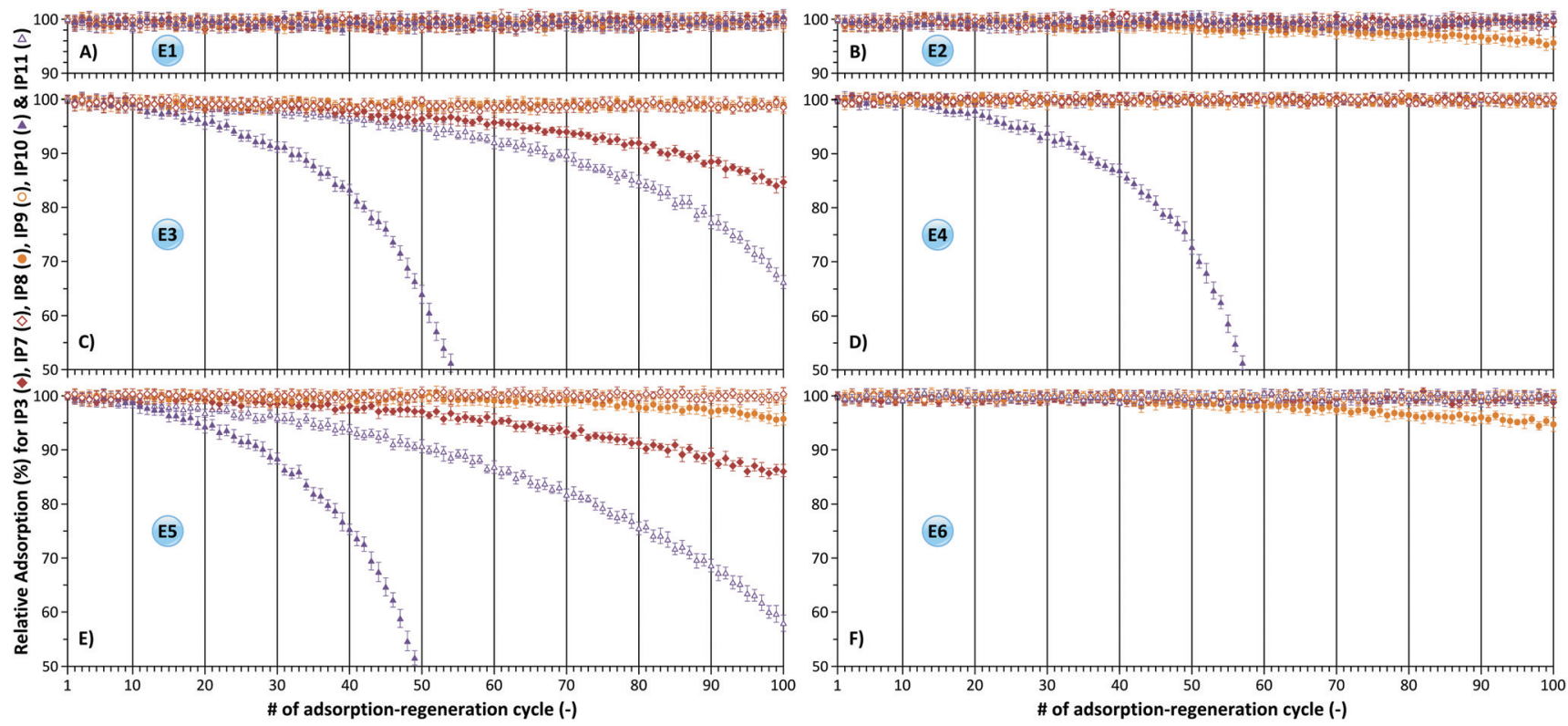

Fig. 8 Relative adsorption of ME on IP3, IP7-11 demonstrating the effect of functional monomers on the reusability of imprinted polymers. Template extraction was carried out using methods E1 (A), E2 (B), E3 (C), E4 (D), E5 (E) and E6 (F) in accordance with Table 2. 
tively removed in order to ensure reliable results, in particular if IPs are used for quantitative analytical applications or product purification. Such clean-up is time-consuming and requires substantial amounts of the extraction solvent. Eppler et al. developed an extraction device specifically designed to facilitate the rapid and efficient clean-up of imprinted polymer matrices offering significant advantages: the ability to use solvent mixtures, temperature-controlled extraction conditions, improved kinetics, and continuous control of the extraction process. ${ }^{16}$ However, both reversible and irreversible degradation of the polymer network can occur under basic and acidic conditions.

EDMA and BMA crosslinked polymers can readily undergo transesterification and hydrolysis under acidic and basic conditions, respectively (Fig. 10A and B). Both reactions result in losing their crosslinking function, which eventually leads to reduced stability and reusability as demonstrated via TGA, SEM, adsorption and selectivity studies. Although the resulting esters or sodium salts remain part of the polymer network, the ethylene glycol and methylenediamine leach out from the adsorbents and could have an adverse effect during the application of the imprinted polymer. Boronic acids can also undergo irreversible esterification in methanol in the presence of acids and the reaction can proceed further resulting in the elimination of trimethyl borate (Fig. 10C), which again leaches out from the adsorbent. On the other hand, esterification of boronic acids is not catalysed by bases and consequently only sodium salt formation can occur, which is reversible
(Fig. 10D). Such behaviour of boronic acids is the reason why extraction methods E3 and E5 result in performance loss of IP10 with regard to adsorption capacity (Fig. 8C and E) and selectivity (Fig. 9), whilst the method E6 incorporating a final acidic wash facilitates maintaining the adsorption (Fig. 8F) and selectivity performance over 100 cycles. Both the esterification and sodium salt formation of carboxylic acids are reversible (Fig. 10E), and thus the performance of IP3 and IP11 can be maintained by methods E4 and E6 (Fig. 8D and F). The decomposition mechanism presented here is in line with the observations on the surface area change over time (Fig. 7). Partial degradation of the crosslinked structure (Fig. 10A and B) results in the collapse of the polymer network decreasing the surface area, while the surface areas for the DVB-based polymer remain virtually constant.

On the other hand, the surface area for polymers with boronic acid functional groups increases over cycles when the extraction method E5 is employed resulting in trimethyl borate elimination (Fig. 10C). The DVB crosslinked polymer network is not affected by the extraction, but the binding sites change, which ultimately results in the increase of the surface area without collapse of the 3D polymer network.

It has been demonstrated that apart from template and monomer leaching, polymer degradation can also result in contamination of samples during the application of IPs. Consequently, both the monomers and the conditions for adsorbent regeneration need to be carefully selected.

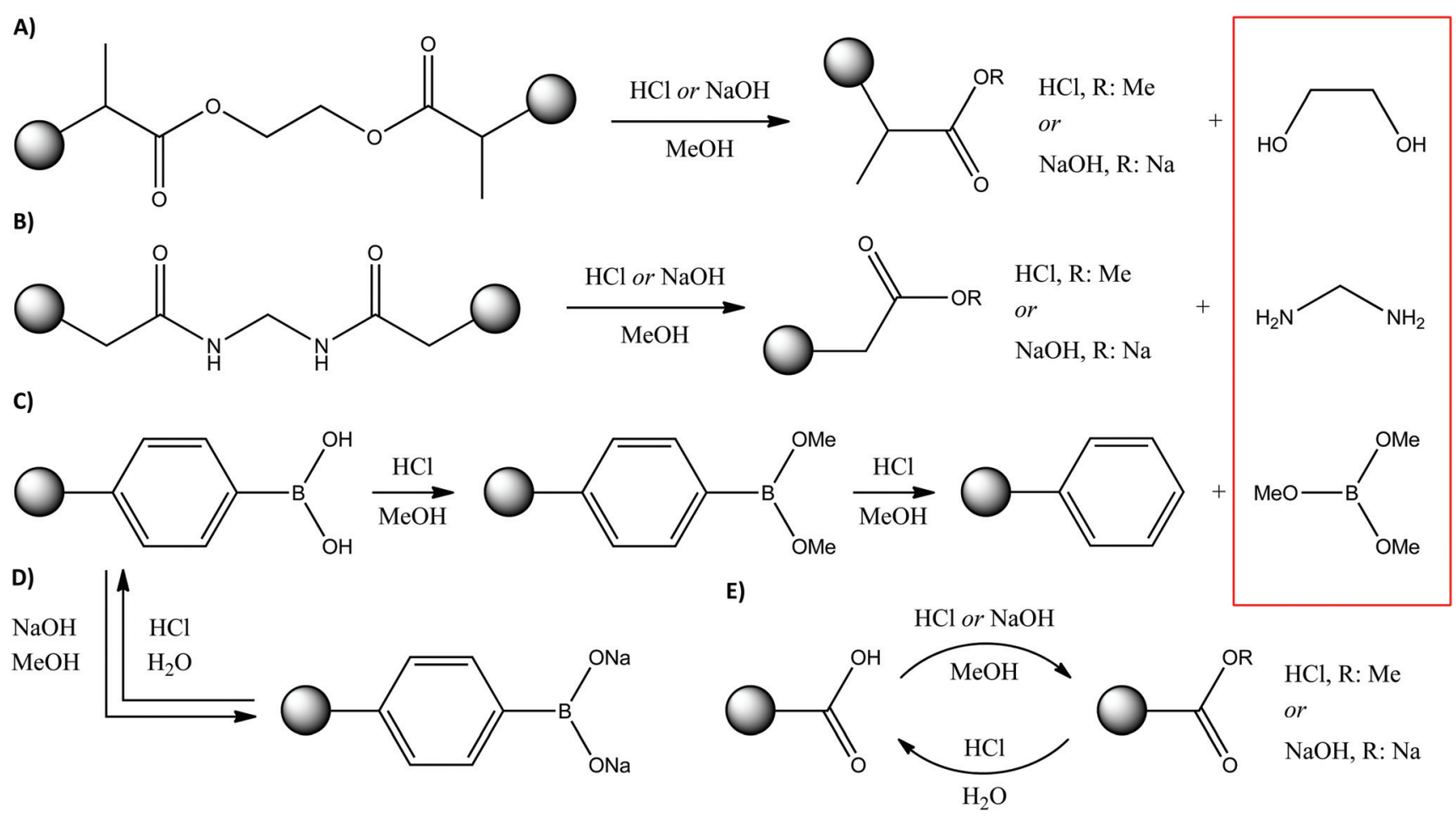

Fig. 10 Degradation schemes of EDMA (A), BMA (B) crosslinked polymers, and esterification of acid based functional monomers via hydrolysis in methanol $(C-E)$. The resulting esters or sodium salts remain part of the polymer network whilst ethylene glycol (A), methylenediamine (B) and trimethyl borate (C) leach out from the adsorbents (see in the box). The elimination by-products were isolated and their characterisation can be found in the ESI. $\dagger$ 


\section{Conclusions}

The reusability of imprinted polymers has a crucial role in developing applications that are reliable, economic and sustainable. The effect of crosslinkers, functional monomers and conditions for template extraction was systematically investigated to reveal the long-term stability and reusability of imprinted polymers. Adsorption-regeneration cycles were carried out 100 times for L-phenylalanine methyl ester imprinted polymers. Irrespective of the degree of crosslinking, divinylbenzene-based polymers demonstrated the most robust behaviour compared to methacrylate- and acrylamide-based polymers. These polymers can be reused at least 100 times without loss of affinity towards the template molecule under acidic and basic conditions, and an elevated temperature $\left(65^{\circ} \mathrm{C}\right)$. In contrast, the performance of methacrylate- and acrylamide-based polymers deteriorates over time under both acidic and basic extraction conditions. It has been demonstrated that besides the well-known template and monomer leaching, polymer degradation products can also result in contamination of samples during the application of the adsorbents. Performance loss of imprinted polymers having carboxylic acid functional moieties can be recovered by an aqueous acidic wash at the end of the regeneration cycle. However, methacrylateand acrylamide-based polymers irreversibly degrade under both acidic and basic conditions, which results in decreased degree of crosslinking. Imprinted polymers having a boronic acid functionality should not be regenerated under acidic conditions in alcohol as it results in their irreversible degradation and elimination of trialkyl borates. Further challenges to be resolved include the regeneration of macromolecularly imprinted polymers. ${ }^{26}$

\section{Acknowledgements}

Useful discussions and analytical insights provided by Peter Pogany from Gedeon Richter Plc are greatly acknowledged. The authors thank Patrick Hill and Christos Didaskalou for their technical assistance with SEM analysis. The financial support for RK, SB and GS through the Royal Academy of Engineering's Newton Research Collaboration Programme under grant code NRCP1516/1/41 is greatly acknowledged. The financial support for JK through the Hungarian Scientific Research Fund/ National Research Development Office, Hungary (OTKA/NKFIH no. PD108462) is acknowledged. MR and GS would like to acknowledge the funding under grant reference 'BioProNET BIV Nov15 Szekely' from BioProNET, a BBSRC Network in Biotechnology and Bioenergy, co-sponsored by the EPSRC.

\section{Notes and references}

1 M. J. Whitcombe, N. Kirsch and I. A. Nicholls, J. Mol. Recognit., 2014, 27, 297.
2 B. Sellergren and C. J. Allender, Adv. Drug Delivery Rev., 2005, 57, 1733.

3 X. Shen, C. Xu and L. Ye, Ind. Eng. Chem. Res., 2013, 52, 13890.

4 M. Ulbricht, J. Chromatogr., B: Biomed. Appl., 2004, 804, 113.

5 Molecularly Imprinted Sensors - Overview and Applications, ed. S. Li, Y. Ge, S. A. Piletsky and J. Lunec, Elsevier, 1st edn, 2012, ISBN 9780444563330.

6 Molecularly Imprinted Catalysts - Principles, Syntheses, and Applications, ed. S. Li, S. Cao, S. A. Piletsky and A. P. F. Turner, Elsevier, 1st edn, 2015, ISBN 9780128013014.

7 A. Bossi, F. Bonini, A. P. F. Turner and S. A. Piletsky, Biosens. Bioelectron., 2007, 22, 1131.

8 X. W. Fu, Q. Yang, Q. Zhou, Q. Lin and C. Wang, Open J. Org. Polym. Mater., 2015, 5, 58.

9 D. Spivak, M. A. Gilmore and K. J. Shea, J. Am. Chem. Soc., 1997, 119(19), 4388.

10 P. Pogany, M. Razali and G. Szekely, Spectrochim. Acta, Part A, 2017, 170, 69.

11 E. Piletska, V. Karim, M. Cutler and S. A. Piletsky, J. Sep. Sci., 2013, 36, 400.

12 E. Piletska, J. Kumire, T. Sergeyeva and S. Piletsky, J. Chin. Adv. Mater. Soc., 2013, 1(3), 229.

13 J. Svenson and I. A. Nicholls, Anal. Chim. Acta, 2001, 435, 19.

14 A. Ellwanger, C. Berggren, S. Bayoudh, C. Crecenzi, L. Karlsson, P. K. Owens, K. Ensing, P. Cormack, D. Sherrington and B. Sellergren, Analyst, 2001, 126, 784.

15 R. A. Lorenzo, A. M. Carro, C. Alvarez-Lorenzo and A. Concheiro, Int. J. Mol. Sci., 2011, 12, 4327.

16 S. Eppler, M. Stegmaier, F. Meier and B. Mizaikoff, Anal. Methods, 2012, 4, 2296.

17 P. Humphries, E. Pretorius and H. Naude, Eur. J. Clin. Nutr., 2008, 62, 451.

18 S. 1. Burgert, D. W. Andersen, L. D. Stegink, H. Takeuchi and H. P. Schedl, Metabolism, 1991, 40(6), 612.

19 A. J. Hall, P. Manesiotis, M. Emgenbroich, M. Quaglia, E. De Lorenzi and B. Sellergren, J. Org. Chem., 2005, 70, 1732.

20 J. Kupai, E. Rojik, P. Huszthy and G. Szekely, ACS Appl. Mater. Interfaces, 2015, 7(18), 9516.

21 R. Haefele, New polymeric reversed-phase column with improved solvent stability and low bleed characteristics, in The Application Notebook, LCGC, 2006.

22 N. Panyachariwat and H. Steckel, J. Soc. Cosmet. Chem., 2014, 65, 187.

23 Z. Ren, D. Kong, K. Wang and W. Zhang, J. Mater. Chem. A, 2014, 2, 17952.

24 A. Venkatesh, N. Chopra and R. J. Krupadam, Environ. Sci. Pollut. Res., 2014, 21, 6603.

25 J. He, A. Liu and J. P. Chen, J. Colloid Interface Sci., 2015, 439, 162.

26 Z. Iskierko, P. S. Sharma, K. Bartold, A. Pietrzyk-Le, K. Noworyta and W. Kutner, Biotechnol. Adv., 2016, 34(1), 30. 